\title{
A Methodology for Mapping Launch Vehicle Buffet Loads
}

\author{
Jordan B. Schwarz ${ }^{1}$ \\ Qualis Corporation, Huntsville, Alabama, 35805
}

\begin{abstract}
Buffet loads represent the primary source of high frequency loading for launch vehicles during the ascent portion of flight. Currently, experimental techniques establish the nature of buffeting using a rigid scale model of the vehicle. The buffet forcing functions resulting from such tests are then applied to reduced finite-element models of the full-scale vehicle to determine the response and consequent loading. This paper discusses the techniques required to translate model-derived, empirical buffet forcing functions into responses for the full-scale launch vehicle, as used to determine the buffet loading for NASA's Ares I launch vehicle.
\end{abstract}

\section{Introduction}

$\mathrm{B}_{\mathrm{r}}^{\mathrm{v}}$ UFFET loads have been a key consideration for launch vehicles since the earliest years of the U.S. space program. Indeed, launch vehicles have been lost due to the high-frequency vibrations and loading that may occur due to buffet. Buffeting is closely associated with the transonic time of flight, where oscillating shocks and regions of unsteady, separated flow produce random aerodynamic inputs on a vehicle over a wide range of frequencies. This results in excitation of a variety of modes ranging from the vehicle's primary bending modes to higher-frequency component and panel modes. ${ }^{1}$

In the present state of the art, buffet loads are predicted empirically via the measurement of scaled buffet loads on a model. The phenomena associated with buffet are too complicated to be accurately captured via CFD. Buffet is sensitive to the specific geometry of individual launch vehicles, with features such as geometric transitions and protuberances strongly influencing buffet loads. Therefore, in experimental testing it is critical that the geometry being tested accurately reflect that of the actual vehicle.

"Hammerhead" configurations - such as that used by NASA's Ares I vehicle - can lead to severe buffeting. Hammerhead vehicles feature a large-diameter forebody such as a payload shroud followed by a slender afterbody, usually the core of the launch vehicle itself. Flow separation occurs where the body transitions from the enlarged front end to the smaller diameter, leading to buffeting. On Ares I, one such region is located close to the middle of the vehicle near the anti-node of the first bending mode. ${ }^{2}$ These characteristics make buffet loads a significant consideration in the overall vehicle loads for Ares I.

For the NASA Ares I launch vehicle, a rigid buffet test was conducted at NASA Langley's Transonic Dynamics Tunnel. $^{2}$ Here an instrumented, sting-mounted model of Ares I in its first-stage flight configuration was rigidly mounted in the wind tunnel. Flows over a range of velocities and dynamic pressures simulated the conditions experienced by the vehicle in the transonic and low supersonic flight regimes.

In the 3.5\% Ares I Rigid Buffet Model test, 256 pressure transducers monitored pressures at discrete locations over the surface of the model, with sensors concentrated in regions of geometric transition. These unsteady pressures were then integrated over effective areas to obtain the net unsteady force acting on the rigid buffet model in the axial and lateral directions. Via Strouhal scaling, these model-scale forcing functions were converted to full-scale equivalents for use in modeling the Ares I vehicle buffet loads. These scaling relationships can be found in a report on a rigid buffet test on a hammerhead version of the Atlas-Centaur I vehicle. ${ }^{3}$

The time-varying buffet forcing functions from NASA Langley were then applied to a flexibility model in order to determine the buffet response. For the Ares I buffet analysis, a model of the integrated vehicle was provided by NASA's Marshall Space Flight Center. This model, based on finite element representations, was reduced via a CraigBampton reduction.

1 Engineer. 5000 Bradford Dr. Suite B. AIAA Member 247462.

Formerly of Dynamic Concepts, Inc., 6700 Odyssey Dr. Suite 202, Huntsville, AL 35806.

1

American Institute of Aeronautics and Astronautics 
To conduct a buffet analysis, buffet forcing functions are applied to the model's degrees of freedom, and the forced response of the model is determined. In the case of this analysis, experimentally-derived buffet forcing functions were provided at discrete stations based on the geometry of wind tunnel model used; these stations differed from the force application points in the finite element model. This necessitated the development of a mapping between the two geometries. Several mapping methodologies were studied for the Ares I buffet analysis, and the resulting impacts of these mapping choices on the vehicle buffet loads are presented.

\section{Mapping Methodologies}

To determine the dynamic response of Ares I to buffeting, buffet forcing functions must be applied to the flexibility model's force application stations. These stations are physical locations (predominantly on the centerline of the vehicle) that have been retained in the model reduction specifically as load-application points. In this case, the buffet forcing functions were provided at points taken from the location of sensors and pressure taps on the wind tunnel model. These points did not coincide with the force application stations of the flexibility model, so it was necessary to construct a mapping in order to apply the forcing functions to the model.

In order to preserve the fidelity of the buffet forcing functions, it is important that the selected mapping conserve forces and moments. If the buffet loads were purely static, these criteria would be adequate. However, since the buffet forcing functions are provided in the form of time histories, it is also important to consider the phase relationships involved when two forcing functions are loaded at the same location.

This section develops several different techniques for mapping buffet forcing functions to the finite element model. Section IV presents results for buffet loads obtained using these different methods.

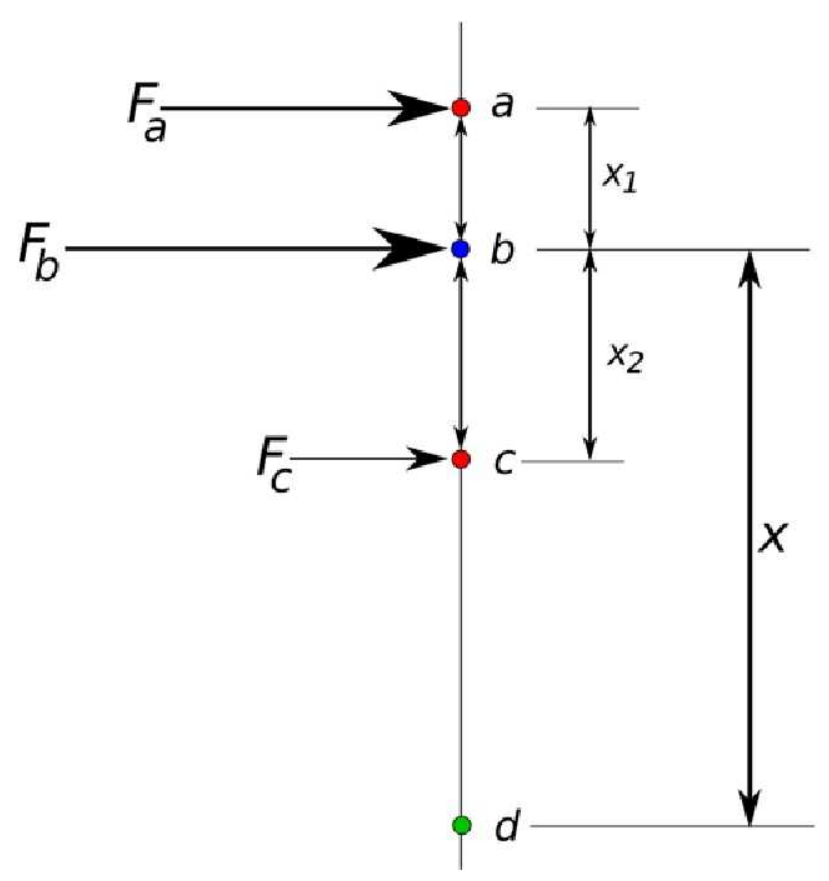

Figure 1. Two-point buffet forcing function mapping. $A$ forcing function at point $\mathrm{b}$ can be mapped to flexibility model stations at a and $\mathrm{c}$, provided that the force mapping conserves forces and moments.

\section{A. Two-Point Mapping}

The most basic mapping technique transfers loads to points on flexibility model and conserves forces and moments but makes does not preserve phasing in doing so. This method is exact for the static case, however. A buffet forcing function cannot be transferred to a single point on the Ares I model while still conserving moments, so it is necessary to apply the load to two points with appropriate weights so that the moment is balanced.

Consider the schematic seen in Figure 1. A force, $F_{b}$ is applied at some generic Buffet Forcing Function (abbreviated here as BFF) station $b$. This force can be converted to a pair of forces applied at stations $a$ and $c$ that when summed equal the original force. The net moment applied by this pair of forces must be the same as that generated by $F_{b}$. This will be the case if the net torque generated by $F_{a}$ and $F_{c}$ about point $b$ is zero. 
The following choices for $F_{a}$ and $F_{c}$ satisfy the force and moment conservation requirements.

$$
\begin{aligned}
& F_{a}=F_{b}\left(\frac{x_{2}}{x_{1}+x_{2}}\right) \\
& F_{c}=F_{b}\left(\frac{x_{1}}{x_{1}+x_{2}}\right)
\end{aligned}
$$

This weighting satisfies the force summation requirement since

$$
\begin{gathered}
F_{a}+F_{c}=F_{b}\left(\frac{x_{2}}{x_{1}+x_{2}}\right)+F_{b}\left(\frac{x_{1}}{x_{1}+x_{2}}\right) \\
F_{a}+F_{c}=F_{b}\left(\frac{x_{1}+x_{2}}{x_{1}+x_{2}}\right)
\end{gathered}
$$

Therefore, the two component forces sum to equal the magnitude and direction of the original:

$$
F_{a}+F_{c}=F_{b}
$$

By choosing weighting factors based on the moment arms of the component forces, a net torque of zero is produced about point $b$. Taking counter-clockwise moments to be positive, the moment created by $F_{a}$ about point $b$ is $M_{a}=-F_{a} x_{1}$, and that due to $F_{c}$ is $M_{c}=F_{c} x_{2}$. The sum of the moments is therefore $M_{b}=F_{c} x_{2}-F_{a} x_{1}$. Expanding the expressions for the point forces, it is shown that there is no net moment generated about point $b$.

$$
M_{b}=F_{b}\left(\frac{x_{1}}{x_{1}+x_{2}}\right) x_{2}-F_{b}\left(\frac{x_{2}}{x_{1}+x_{2}}\right) x_{1}=0
$$

Furthermore, it can be shown via a similar argument that the moment created by the mapped forces about an arbitrary point $d$ is also conserved. The moment about $d$ created by $F_{b}$ is $M_{b}=-F_{b}(x)$. The combined moment due to $F_{a}$ and $F_{c}$ is $M_{a c}=-F_{a}\left(x+x_{1}\right)-F_{c}\left(x+x_{2}\right)$. Substituting the expressions for $F_{a}$ and $F_{c}$, the moment can be written as

$$
M_{a c}=-F_{b}\left(\frac{x_{2}}{x_{1}+x_{2}}\right)\left(x+x_{1}\right)-F_{b}\left(\frac{x_{1}}{x_{1}+x_{2}}\right)\left(x-x_{2}\right)
$$

Combining the like terms yields

$$
\left(x_{1}+x_{2}\right)\left(\frac{M_{a c}}{-F_{b}}\right)=x_{2}\left(x+x_{1}\right)+x_{1}\left(x-x_{2}\right)
$$

Expanding the distributed terms produces

$$
\begin{gathered}
\left(x_{1}+x_{2}\right)\left(\frac{M_{a c}}{-F_{b}}\right)=x x_{2}+x_{1} x_{2}+x x_{1}-x_{1} x_{2} \\
\left(x_{1}+x_{2}\right)\left(\frac{M_{a c}}{-F_{b}}\right)=x\left(x_{1}+x_{2}\right) \\
\left(\frac{M_{a c}}{-F_{b}}\right)=x
\end{gathered}
$$

Finally, this result is identical to the expression for the moment about point $d$ due to a single force at $b$. 


\section{B. Two-Point Mapping with Correlation Minimization}

While the two-point mapping ensures the conservation of forces and moments between the two sets of coordinates, the method makes no corrections for phase cancellations which may occur when multiple time-varying loads are combined at a single point. It was suggested that if multiple forcing functions were combined in a way that minimized the correlation between them, this would reduce the potential phase-canceling effects of this method. $^{4}$

In general, phasing of the forcing functions presents the greatest concern when coupling occurs between the fluid and structural systems. However, in the case of the Ares I Rigid Buffet Model, it is expected that any structural interaction will be eliminated via the use of a rigid model. As a result, the correlation of the forcing functions along the length of the vehicle is already quite low, with correlation coefficients in the range of 0.1 or lower.

The full-scale Ares I buffet forcing functions used in this analysis are each about 433 seconds in length. Previous temporal convergence studies have shown that only a 30-60 second window of each forcing function is needed to obtain sufficient convergence of the buffet response, and a 30 -second transient provides very good convergence with substantially reduced computational overhead.

As a result, it is possible to pick the "windows" appropriately to minimize the correlation between summed forcing functions. A MATLAB program was designed that partitioned each buffet forcing function into 30 -second windows, then picked forcing function blocks in a way that minimized the correlation. The first forcing function assigned any point is unique and can be chosen from an arbitrary window. When summing additional forcing functions, the program computes the correlation between the existing forcing function and all available 30 -second windows for the new forcing function. The program then chooses the block that minimizes correlation. Using this method, the correlation coefficients were reduced by an order of magnitude to near zero.

\section{Four-Point Mapping}

The preceding two-point mapping methodology transfers loads to pairs of points in the new coordinate frame. It was suggested that mapping the buffet forcing functions to greater numbers of points would yield more accurate results by expanding the range of influence of each forcing function, distributing the function over more stations on the finite element model. ${ }^{4} \mathrm{~A}$ four-point mapping method was developed in order to validate this suggestion.

In this method, each buffet forcing function in the source coordinate frame is mapped to four points in the new frame, bracketed by two points on each side as shown in Figure 2.

The four-point method essentially splits force $F_{b}$ into two forces, then uses the twopoint method to apply part of the force at $b$ to an inner pair. The remainder of the force is applied to a second, outer pair of points, again using the two-point method. Since the force and moment are conserved for each instance of the two-point method, then the total force and moment are also conserved in

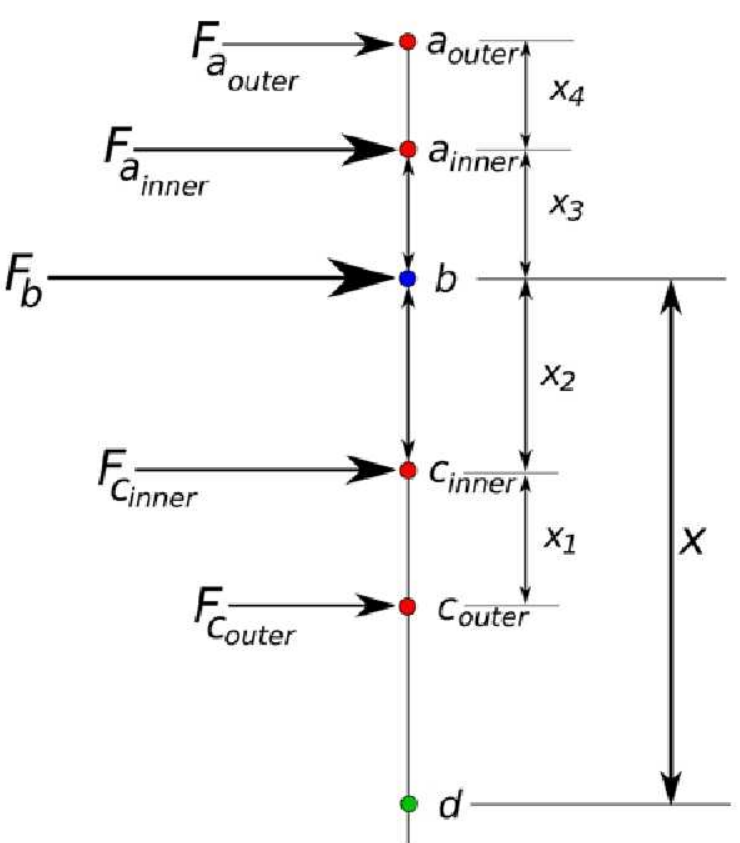

Figure 2. Four-point buffet forcing function mapping. $A$ forcing function at point $\mathrm{b}$ can be mapped to four flexibility model stations instead of two. this implementation of the four-point method.

This method is effectively a cascade of two-point mapping elements. In principle, more stages could be added to the cascade to further distribute buffet forcing functions. However, as the cascade becomes more complicated, it becomes increasingly difficult to trace the influence of an experimental forcing function on the stations of the finite element model. 
Although the four-point method has greater complexity than the two-point method, it is possible to use an identical correlation-minimizing algorithm. One possible advantage to this method is that each buffet forcing function can be assigned a larger region of influence than with the two-point method, and the weights of the "inner" and "outer" sets of forces can be tailored to achieve the desired force distribution. Note also that typically the weights applied to the mapped forces sum to unity; this ensures that forces and moments are conserved. If for some reason a non-conservative force distribution is desired, it is possible to use weights with a sum greater than one. The impacts of such weighting choices were studied, and these results are presented in Section IV of this paper.

\section{Buffet Analysis Methodology}

The Ares I buffet analysis made use of multiple finite element-based flexibility models corresponding to discrete times of flight. These models were similar structurally but differed principally in their mass distribution due to fuel burn. The entire phase of $1^{\text {st }}$ stage ascent was divided into a series of analysis periods, each utilizing a corresponding, stationary mass model. Previous studies have indicated the validity of this technique, provided that the periods are sufficiently short in duration. ${ }^{5}$

For a comprehensive buffet loads analysis, buffet would be modeled for a selection of representative trajectories and dynamic pressure $(q)$ values. This technique is used to estimate the variation of flight loads with trajectory and flight conditions. However, this analysis of forcing function mappings studied only a single reference trajectory family. This approach allowed the impacts of the forcing function mapping methods to be assessed while keeping trajectory-dependent variables constant. Four analysis periods were created, spanning the times of flight where buffet loading typically occurs. The parameters assigned to each analysis period are shown in Table 1.

\begin{tabular}{|c|c|c|c|c|}
\hline Period & Center Mach No. & Condition & Finite Element Model & Buffet Forcing Function \\
\hline 1 & 0.7 & Transonic Flight & $t=40$ model & $M=0.82, \alpha=4 \mathrm{deg}$ \\
\hline 2 & 1.05 & Transonic Flight & $t=40$ model & $M=0.98, \alpha=4 \mathrm{deg}$ \\
\hline 3 & 1.4 & $\operatorname{Max} q-\alpha$ & $t=50$ model & $M=1.20, \alpha=4 \mathrm{deg}$ \\
\hline 4 & 1.8 & $\operatorname{Max} q$ & $t=60$ model & $M=1.55, \alpha=4 \mathrm{deg}$ \\
\hline
\end{tabular}

Table 1: Description of run conditions for buffet mapping analysis.

To determine the buffet loads for each analysis period, a 30-second window from the buffet forcing function was applied to the model. A modal transformation was applied to the model, and modes above $60 \mathrm{~Hz}$ were truncated. This truncation is a standard practice, because buffet forcing functions contain content to only about $50 \mathrm{~Hz}$, and the higher vehicle modes in the finite element model cannot generally be trusted. High frequency buffet tends to excite components rather than the vehicle modes, and different modeling techniques are required to adequately capture buffet loading.

The modal system was then integrated using a fourth order Runge-Kutta method. Following a transformation of the integrated response back to the original set of coordinates, the centerline forces and moments were recovered through the use of load transformation matrices (provided with the finite element models). The entire buffet analysis process was implemented as a series of MATLAB scripts.

Note that when examining buffet loads, the key parameter is typically taken to be the RMS level of the unsteady buffet loading, scaled to the appropriate level of enclosure. Buffet loads follow a Rayleigh distribution rather than a normal (Gaussian) distribution; consequently, buffet loads are not reported in terms of the standard deviation.

\section{Results}

The impacts of the different buffet forcing function mapping techniques may be observed through their effects on forcing function RMS levels at locations over the length of the Ares I vehicle. Figures 3-5 compare the centerline RMS force levels for the various mapping techniques with the nominal levels of the original, unmapped forcing functions as these exist in the wind tunnel model coordinates. A linear interpolation was performed to convert load levels in the wind tunnel model frame to the coordinates used by the finite element model. Only data from the $M=0.98, \alpha=4$ degree case are shown, as trends for the other Mach numbers are very similar.

Note the discrepancies observed between the interpolated original data set and the mapped forcing functions. An ideal mapping would preserve the magnitude of the original forces, resulting in a zero-percent difference between 
the mapped force and the original. However, significant differences in load levels arise because the forcing functions are distributed to a much larger number of points in the new coordinate frame. Large differences are also seen when the original and mapped locations of forcing functions are far apart. These numerical issues, indicate that some discrepancy will inevitably exist between any mapped forcing function and its original counterpart. Therefore, the usefulness of Figures 3-5 lies in the relative trends and differences observed between mapping methods.

For the two-point methods, little improvement is seen in results due to use of correlation reduction algorithms. Since the buffet forcing functions are relatively uncorrelated to begin with, this trend is not surprising.

For the axial buffet forcing functions seen in Figure 3, it is clear that the 4-point uncorrelated method results in RMS levels typically lower than those of the 2-point methods. This is especially true for locations near the Ares I crew module, a region of major interest with regard to buffet loads.

The impacts of the mapping methods on lateral forcing functions are shown in Figures 4 and 5. The distribution of lateral buffet forcing functions is relatively continuous along the vehicle, while the axial stations are concentrated only at regions of sharp geometry change, which contribute significantly to axial buffeting. As in the axial case, the 4-point method is seen to result in lower loads than the 2-point methods. Use of the correlation reduction techniques does not greatly reduce the applied loads. Figure 4 shows the Y-direction results; the Z-direction results seen in Figure 5 have similar trends.

Based on these results, it is observed that the two-point buffet mapping method provides a high-quality mapping as well as a straightforward implementation. It should be noted that these results are specific to buffet forcing functions which are largely uncorrelated. For highly correlated signals, special considerations must be made to preserve the phasing of the transients. In the case of the relatively uncorrelated buffet forcing functions analyzed here, the correlation-reducing methods were found to offer little performance improvement and did not have a significant impact on the buffet forcing function levels.

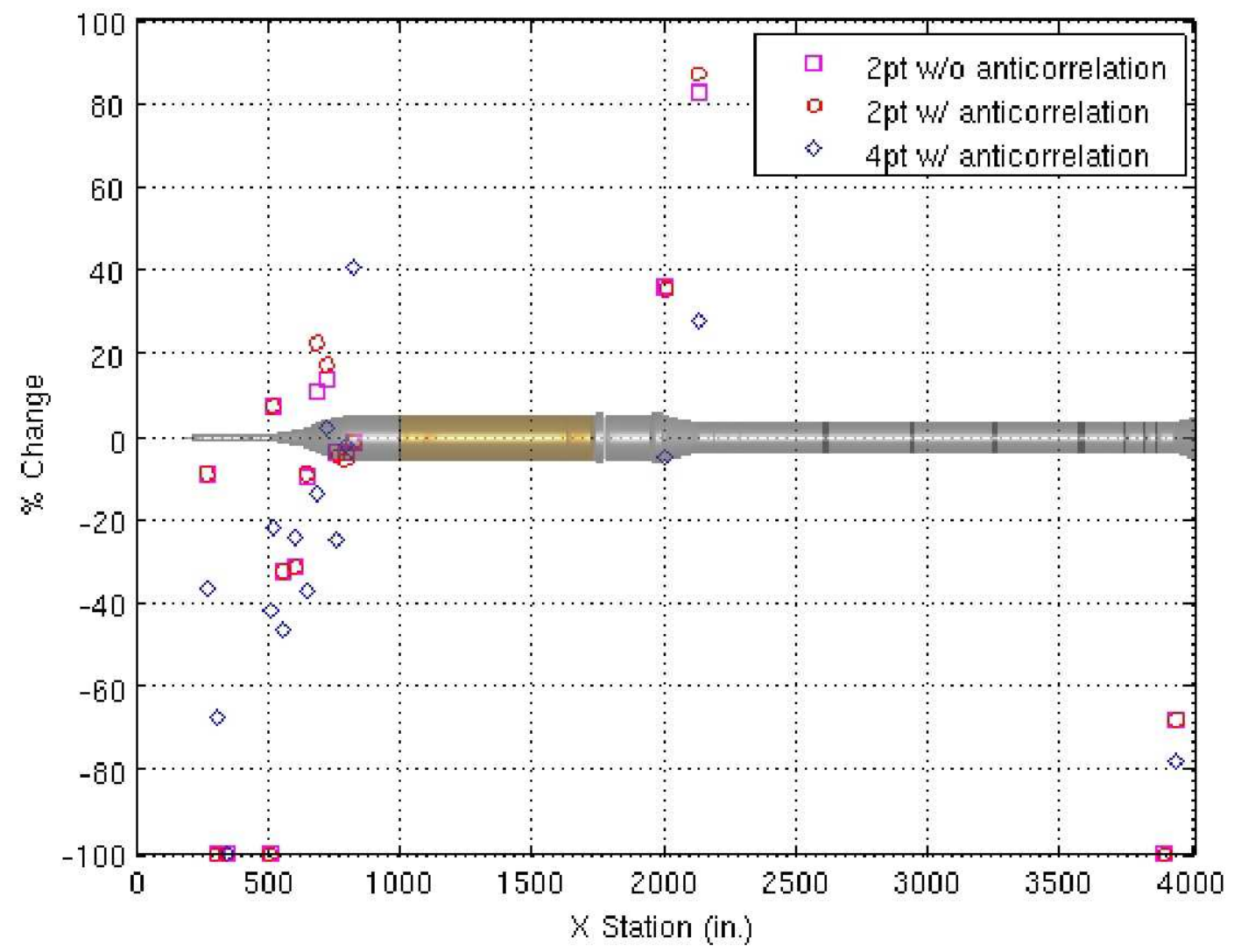

Figure 3. Effect of buffet mappings on axial RMS levels. 


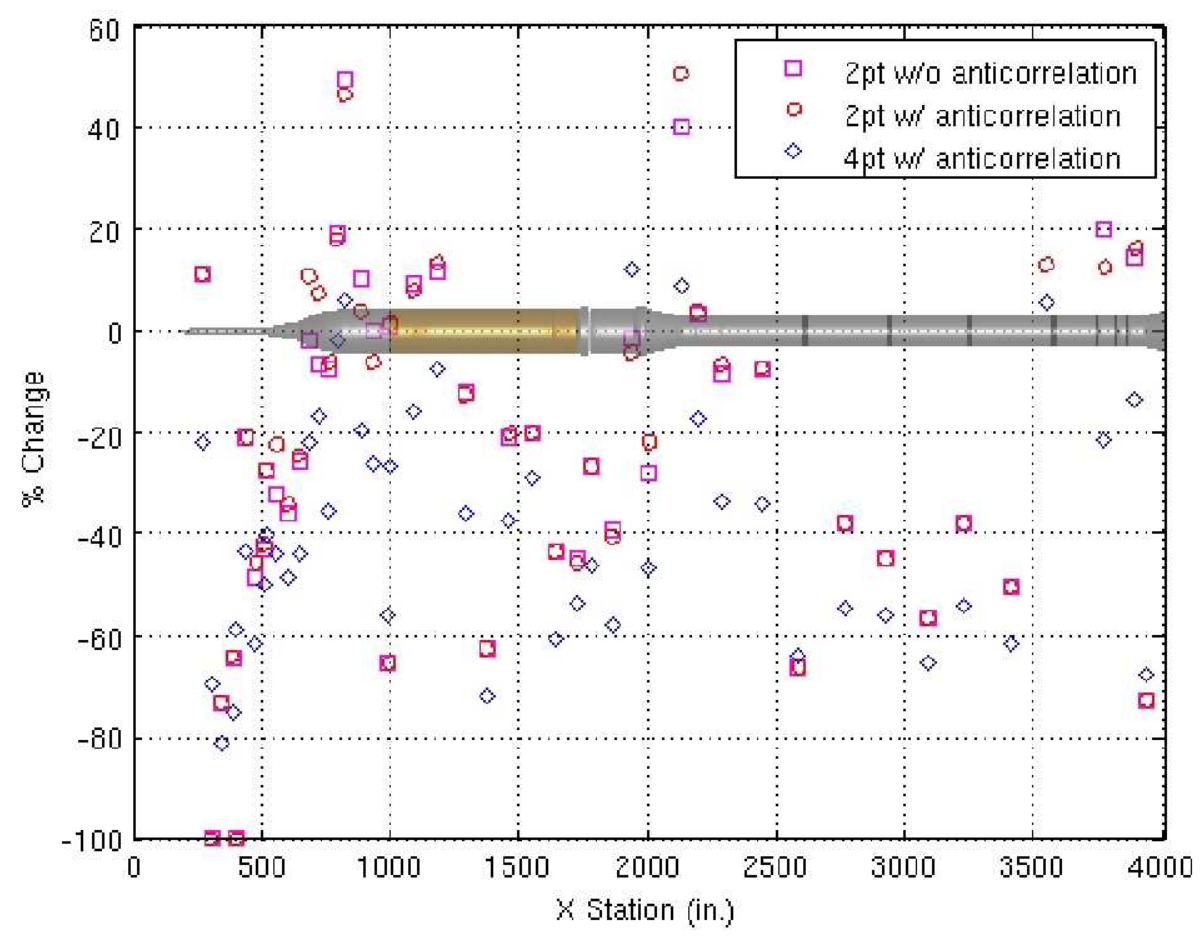

Figure 4. Effect of buffet mappings on lateral RMS levels, $Y$ direction.

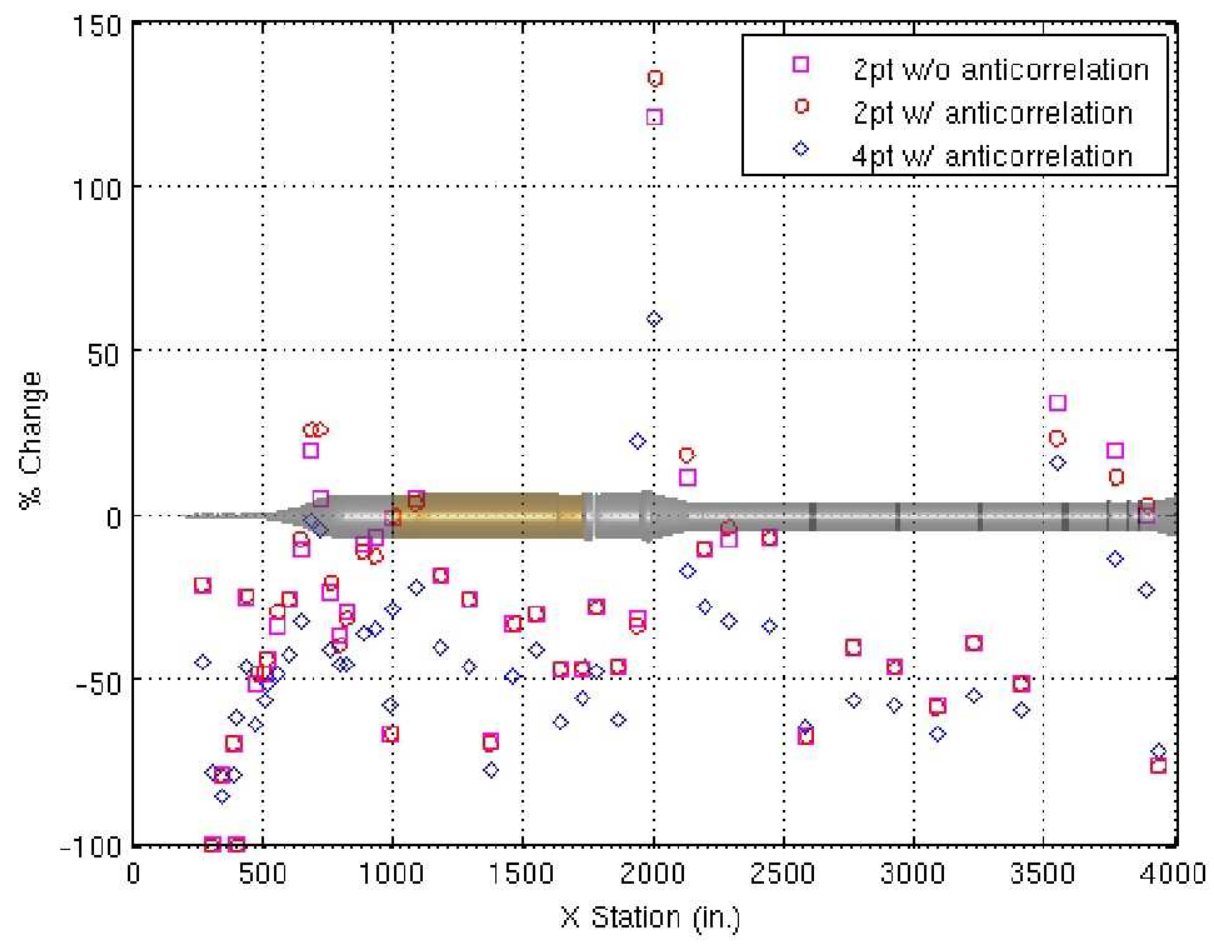

Figure 5. Effect of buffet mappings on lateral RMS levels, $Z$ direction. 


\section{Summary}

This paper developed techniques for mapping buffet forcing functions from one model geometry to another. In the case of the Ares I buffet loads analysis, these methods were used to transfer buffet forcing functions from a wind tunnel model coordinate frame to a finite element model. Analogous techniques could also be used to transfer loads from one finite element to another as models are periodically updated and improved.

Two mapping techniques were developed: a simple, two-point mapping that preserved static force and moment relationships, and a four-point mapping also conserved these quantities while allowing greater tailoring of the buffet force distribution. These techniques do not conserve dynamic forces and moments because of the potential for phase cancellation when dynamic signals are added. Therefore, a technique was developed to minimize the correlation of additive signals, decreasing the likelihood of phase cancellation.

In practice, the buffet forcing functions used in this analysis were found to be sufficiently uncorrelated to begin with; consequently, the correlation reduction method had little effect on buffet force magnitudes. The two-point buffet mapping method was seen to better preserve the character of the original buffet forces. The two-point method maps each buffet force to the minimum number of points needed in the new coordinate frame.

The four-point mapping method led to a somewhat pathological effect: the multiple force distribution locations used in this method resulted in forcing functions dissimilar to their original counterparts. Forces were distributed further from their locations in the original coordinate frame. This issue could be corrected by using a nonconservative force weighting, however this technique was seen to increase buffet loads in a way that could not easily be quantified.

This analysis focused on the effects of mapping on the applied buffet force RMS levels. Future work should also consider the impacts on the resulting buffet loads and accelerations, which are important considerations for any buffet analysis. In addition, mapping effects should be studied in the frequency domain, using the Power Spectral Density of forces, loads, and accelerations to assess any frequency impacts created by the mapping techniques.

\section{References}

\footnotetext{
${ }^{1}$ Cole, H.A., Erickson, A.L., and Rainey, A.G., "Buffeting During Atmospheric Flight," NASA SP-8001, November 1970 revision of May 1964 printing.

${ }_{2}^{2}$ Piatak, D.J. and Sekula, M.K., "Database of Ares I Full-Scale Buffet Forcing Functions," ARES-AE-DBR-0001 R1.0, NASA Langley Research Center, 15 April 2009.

${ }^{3}$ Cole, S.R. And Henning, T.L., "Dynamic Response of a Hammerhead Launch Vehicle Wind Tunnel Model," NASA TM 104050, NASA Langley Research Center, February 1991.

${ }^{4}$ Kabe, A. and Chen, S., "Ares I Buffet Forcing Functions TIM," Huntsville, AL and El Segundo, CA, 7 May 2009.

${ }^{5}$ Dotson, K.W. and Tiwari, S.B., "Formulation and Analysis of Launch Vehicle Maneuvering Loads," AIAA Journal of Spacecraft and Rockets, Vol. 33, No. 6, 1996, pp. 815-821.
} 